\title{
Cranial polyneuropathy as the first manifestation of a severe COVID-19 in a child
}

\author{
Aphaia Roussel ${ }^{1}$, David Germanaud ${ }^{1}$, Yassine Bouchoucha ${ }^{1}$, Naim Ouldali ${ }^{1}$, Meryl \\ Vedrenne-Cloquet ${ }^{2}$, Martin Castelle ${ }^{2}$, and Andre Baruchel ${ }^{3}$ \\ ${ }^{1}$ Robert-Debré Mother-Child University Hospital \\ ${ }^{2}$ Necker-Enfants Malades Hospitals \\ ${ }^{3}$ Robert-Debré Hospital
}

July 23, 2020

\begin{abstract}
We report a rare acute neurological complication associated with COVID-19 in a 6-year-old girl with hematopoietic stem cell transplantation (HSCT) for sickle cell anemia. 21 days after transplant she presented an acute facial diplegia, followed by a swallowing and left sensitive involvement, associated with an MRI T2 hypersignal and gadolinium enhancement of facial and hypoglossal nerves. Fever and severe respiratory symptoms appeared secondarily leading to the diagnosis of COVID-19. This cranial polyneuropathy in a child was inaugural, questioning the typical post-infectious mechanism. Physicians should consider SARS-CoV-2 in patients with cranial nerve abnormalities even without respiratory manifestations or fever.
\end{abstract}

\section{Introduction}

Coronavirus disease 2019 (COVID-19), caused by SARS-CoV-2 has led to a major pandemic in early 2020. The typical clinical syndrome is a respiratory illness, which can be responsible for severe viral pneumonia (1). In infected children symptoms are rather milder compared to adults (2), with a majority of paucisymptomatic forms (3). In severely ill pediatric patients, main symptoms are fever, tachypnea, productive cough, expectoration, nausea/vomiting, diarrhea and asthenia (4). Various neurological symptoms were also reported in adults and children, consistent with a neurological tropism of coronaviruses that receive more and more interest in the case of SARS-CoV-2 (5). We report on a 6-year-old girl with early cranial polyneuropathy associated with COVID-19 after allogeneic hematopoietic stem cell transplantation (HSCT) for sickle cell anemia (SCA).

\section{Case presentation}

A 6 year-old girl with sickle cell anemia complicated by cerebral vasculopathy received hematopoietic stem cell transplantation (HSCT) from a matched-related donor after myeloablative conditioning (Busulfan, $\mathrm{Cy}-$ clophosphamide, Anti-T-lymphocyte globulin) (Figure 1). Graft-versus-host disease (GvHD) prophylaxia included Ciclosporin and Methotrexate. Historical timeline is presented in Figure 1.

Various infectious complications occurred around the grafting date:

- Asymptomatic EBV replication 14 days before the graft leading to the administration of one dose of rituximab infusion.

- Severe mucositis aggravated by HSV-1 infection (oral and pelvic localization) early after conditioning treated with high-dose of Aciclovir. 
- Febrile neutropenia (48 hours) during HSV-1 reactivation without bacterial documentation, was treated with broad spectrum antibiotics (Tazocilline from day 0 to 20 post-transplant, Vancomycine from day 2 to 15 post-transplant and Ciprofloxacine from day 2 to 10 post-transplant).

After engraftment on day 20, antibiotics were stopped, mucositis and signs of HSV1 infection had fully resolved, and IV prophylactic aciclovir was started.

At day 21, she presented with bilateral facial palsy and left trigeminal hypoesthesia and swallowing impairment with voice modification. Cerebral MRI displayed T2 T2-FLAIR hypersignal of the intracranial and meatal segments of the facial nerves, with $\mathrm{T} 1$ Gadolinium-enhancement that also concerned the left hypoglossal nerve, without haemorrhage or cerebral ischemia nor PRES syndrome (Figure 2). First cerebrospinal fluid (CSF) examination was normal, protein level of $25 \mathrm{mg} / \mathrm{dL}$ (reference range 15-60 mg/dL), glucose level of $66 \mathrm{mg} / \mathrm{dL}$, white blood cells 1 per $\mu \mathrm{L}$ (reference range 0-5 per $\mu \mathrm{L}$ ), and without concomitant detected red blood cells. CSF Gram stain and culture were negative, and HSV, VZV, enterovirus, EBV, HHV6, HHV8, adenovirus, mycoplasma, cryptococcus and toxoplasmose PCR were negative. C-Reactive-Protein (CRP) was $<10 \mathrm{mg} / \mathrm{ml}$.

The onset of facial diplegia was rapidly progressive, followed after 24 hours by swallowing impairment. Clinically detectable neurological damage was limited to the cranial nerves $\left(\mathrm{V}^{\text {st }}\right.$, VII ${ }^{\text {st }}$ and IX $^{\text {st }}$ pairs $)$, with no evidence of limb sensory-motor deficit or areflexia. Intravenous immunoglobulins $(1 \mathrm{~g} / \mathrm{kg})$ were infused at day 22 and 23 without improvement. Second CSF examination was performed: protein level remained low at $30 \mathrm{mg} / \mathrm{dL}$, glucose $63 \mathrm{mg} / \mathrm{dL}$, and white blood cells rised at 70 per $\mu \mathrm{L}$ after immunoglobulins.

Fever and respiratory symptoms appeared secondarily at day 25 post-transplant, 4 days after diplegia, with productive cough and fever up to $40^{\circ}$. CRP remained negative. Chest-CT scan showed COVID-19 typical findings. SARS-Cov2 RT-PCR assay was positive in nasopharyngeal and blood, not in CSF. Acute respiratory distress syndrome led to transfer in intensive care unit.

She underwent protective mechanical ventilation and sedation for 17 days and received treatment by Remdesevir (10 days) and Tocilizumab. Neurological status could not be assessed during sedation for mechanical ventilation. Facial diplegia was persistent beyond mechanical ventilation. She received a second infusion of intravenous immunoglobulins at day 51 and 52. She showed significant clinical improvement and had normal nerve conduction study of the limbs when achievable at day 60 .

\section{Discussion}

We present the first description of cranial polyneuropathy involvement in a child infected with SARS-COV-2. Although COVID-19 is mainly associated with a respiratory illness, neurological involvement shall not be underestimated as it has been reported in more than $35 \%$ cases in a recent study of 214 patients (6). Three types of neurological symptoms have been described: (i) central nervous system manifestations as dizziness, headache, impaired consciousness, acute cerebrovascular disease, ataxia, or seizure, (ii) peripheral nervous system manifestations as taste impairment, smell impairment, vision impairment, and neuropathic pain, (iii) and skeletal muscular injury manifestations. Theses neurological manifestations tended to occur early in the illness (median time, 1-2 days), in patients with severe infection, respiratory symptoms of COVID19 developing secondarily as in our observation. Of the 214 patients, only 5 had isolated peripheral nerve damage, 4 of which progressed to severe COVID-19 as in our observation.

Our observation could be considered an atypical case of Guillain-Barré syndrome, with exclusive craniofacial impairment. One case of Guillain-Barré syndrome associated with SARS-CoV-2 was described in a 61 year old woman (7), recently followed by the description of five patients with Guillain-Barré syndrome 5 to 10 days after the onset of COVID-19, 4 of them presenting with facial weakness or diplegia (8). The pathophysiology of this peripheral nerve damage is not yet established and may not be unequivocally related to a per or post infectious immunological mechanism as seen with other infectious agents (9). Indeed, the cranial polyneuropathy we report was clinically inaugural, in a heavily immunocompromised patient. Besides, we did not observed the typical Guillain Barre CSF change with high protein without high cellularity. In any 
case, in our observation as in the Toledano series, the extent of the facial nerves involvement is striking. All these particularities point to the possible involvement of SARS-Cov 2 neurotropism.

Over the years, some coronaviruses have been associated with neurological diseases in animals. Among the five different coronaviruses able to infect humans, at least three strains are neuroinvasive and neurotropic $(10,11)$. Early in 2002 and 2003, studies on the samples from patients with SARS revealed the presence of SARS-CoV particles in the brain, where they were located almost exclusively in the neurons (9). Experimental studies carried out in mice infected with coronavirus have shown that the brain stem might be strongly impacted $(12,13)$ which raises the hypothesis that an impairment of the central nervous system might be involved in the respiratory failure (14). A potential tropism for cranial nerves has also been disccused, especially with the olfactory nerves and bulbs acting as a doorway to the cerebral nervous system upon a primary nasal infection.

In our patient, no pathogen other than SARS-CoV 2 could be detected, despite two lumbar punctures and extensive research. However, it remains possible that viral co-infections (EBV and HSV) interplayed with the coronavirus in the pathogenesis of this polyneuropathy. Moreover, the conditioning, the resulting immunosuppression or the pre-existing cerebral vasculopathy may also have play a role even if this type of cranial polyneuropathy had not been reported as side effect of conditioning nor in sickle cell disease.

In conclusion, peripheral nerve involvement is rarely described in COVID-19 but might predict an aggressive course of the disease, and facial or cranial nerve involvement may be suggestive. By any means, physicians should consider SARS-CoV-2 infection in immunocompromised patients with peripheral nerve abnormalities even without respiratory manifestations or fever. A prompt diagnosis could prevent delayed management and prevention of transmission of the virus.

4. Conflict of Interest: The other authors have no conflicts of interest to disclose.

\section{References}

1. Guan W-J, Ni Z-Y, Hu Y, Liang W-H, Ou C-Q, He J-X, et al. Clinical Characteristics of Coronavirus Disease 2019 in China. N Engl J Med. 28 févr 2020;

2. Su L, Ma X, Yu H, Zhang Z, Bian P, Han Y, et al. The different clinical characteristics of corona virus disease cases between children and their families in China - the character of children with COVID-19. Emerg Microbes Infect. déc 2020;9(1):707-13.

3. Liu W, Zhang Q, Chen J, Xiang R, Song H, Shu S, et al. Detection of Covid-19 in Children in Early January 2020 in Wuhan, China. N Engl J Med. 02 2020;382(14):1370-1.

4. Sun D, Li H, Lu X-X, Xiao H, Ren J, Zhang F-R, et al. Clinical features of severe pediatric patients with coronavirus disease 2019 in Wuhan: a single center's observational study. World J Pediatr WJP. 19 mars 2020;

5. Marc D, Dominique JF, Elodie B, et al. Human Coronavirus: Respiratory Pathogens Revisited as Infectious Neuroinvasive, Neurotropic, and Neurovirulent Agents. CRC Press; [Internet]. 2013:93-122. ed. Disponible sur: https://www.researchgate.net/publication/243457712_Human_coronavirus_respiratory_pathogens_revisited_as_infectious_neuroinvasive_neurotropic_and_neurovirulent_agents

6. Mao L, Jin H, Wang M, Hu Y, Chen S, He Q, et al. Neurologic Manifestations of Hospitalized Patients With Coronavirus Disease 2019 in Wuhan, China. JAMA Neurol. 10 avr 2020;

7. Zhao H, Shen D, Zhou H, Liu J, Chen S. Guillain-Barre syndrome associated with SARS-CoV-2 infection: causality or coincidence? Lancet Neurol. 1 avr 2020;

8. Toscano G, Palmerini F, Ravaglia S, Ruiz L, Invernizzi P, Cuzzoni MG, et al. Guillain-Barre Syndrome Associated with SARS-CoV-2. N Engl J Med. 17 avr 2020; 
9. Arbour N, Day R, Newcombe J, Talbot PJ. Neuroinvasion by human respiratory coronaviruses. J Virol. oct 2000;74(19):8913-21.

10. Gu J, Gong E, Zhang B, Zheng J, Gao Z, Zhong Y, et al. Multiple organ infection and the pathogenesis of SARS. J Exp Med. 1 aout 2005;202(3):415-24.

11. Li Y-C, Bai W-Z, Hashikawa T. The neuroinvasive potential of SARS-CoV2 may play a role in the respiratory failure of COVID-19 patients. J Med Virol. 27 fevr 2020;

12. Netland J, Meyerholz DK, Moore S, Cassell M, Perlman S. Severe acute respiratory syndrome coronavirus infection causes neuronal death in the absence of encephalitis in mice transgenic for human ACE2. J Virol. aout 2008;82(15):7264-75.

13. Li K, Wohlford-Lenane C, Perlman S, Zhao J, Jewell AK, Reznikov LR, et al. Middle East Respiratory Syndrome Coronavirus Causes Multiple Organ Damage and Lethal Disease in Mice Transgenic for Human Dipeptidyl Peptidase 4. J Infect Dis. 1 mars 2016;213(5):712-22.

14. Desforges M, Le Coupanec A, Stodola JK, Meessen-Pinard M, Talbot PJ. Human coronaviruses: viral and cellular factors involved in neuroinvasiveness and neuropathogenesis. Virus Res. 19 dec 2014;194:145-58.

\section{Figure legends:}

Figure 1 . Scheme presenting the medical history of the patient under study.

BMT: bone marrow transplantation

ICU: Intensive Care Unit

Ig IV: intrevenous immunoglobulins

Figure 2 . Axial slices of brain MRI (T1w TSE SPIR images). Cranial nerves enhancement upon gadolinium injection is shown: blue arrow pointing the hypoglossal nerve (left panel) and the two red arrows pointing the two facial nerves (right panel).

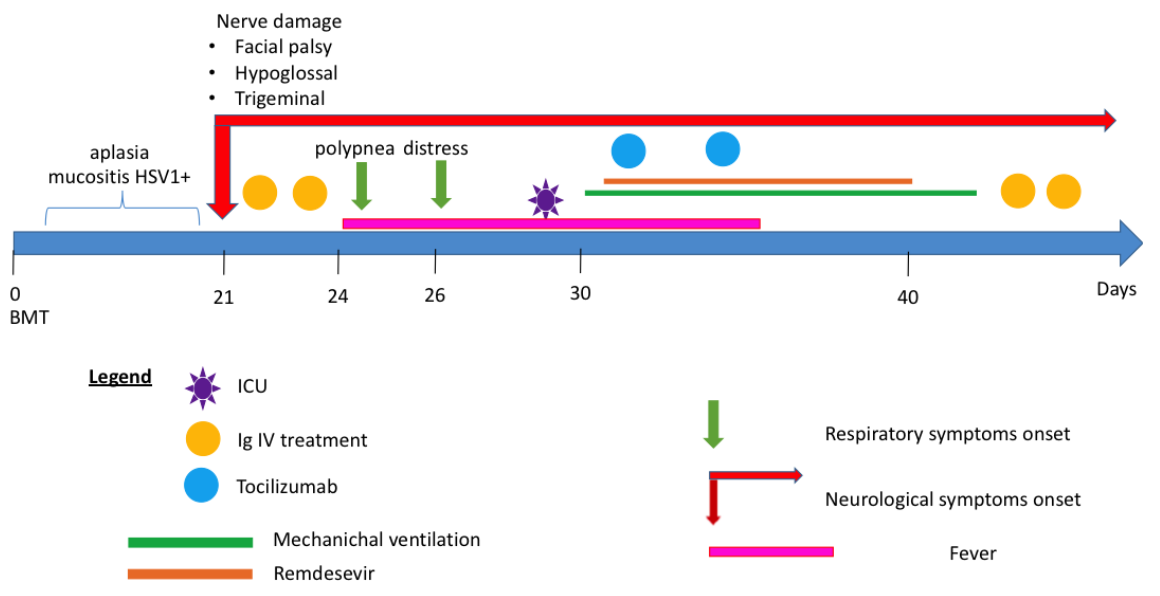



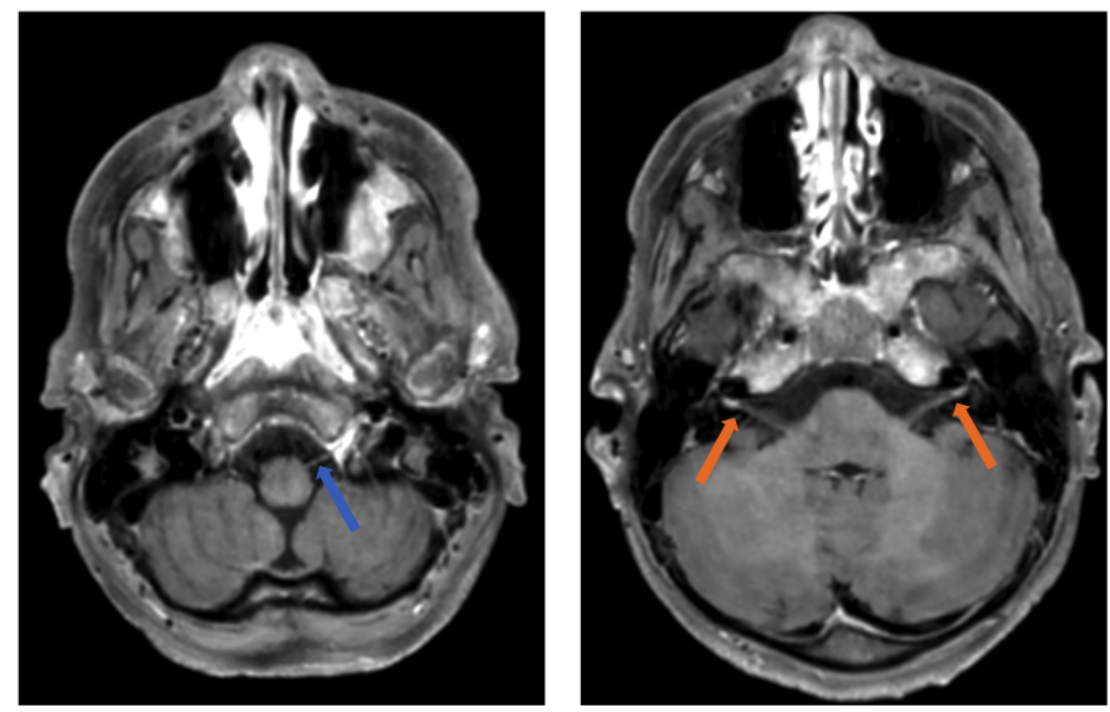